\title{
Sprachpuristische Bestrebungen im 19. Jahrhun- dert in Bezug auf die Jahnsche Turnsprache
}

\author{
Aleksandra Wróbel
}

\begin{abstract}
Linguistic purism in the 19th century in relation to Jahn's gymnastics language Abstract

Turnvater Jahn wanted to make both of gymnastics movement and the gymnastics language at the same time understandable and accessible to everyone. Jahn endeavoured in many ways to enrich and purify the German language as an expression of national consciousness. Every word had to clarify the national spirit and sense of the gymnastic movement in order to contribute to the unification of Germany. Language was the unifying bond of the fragmented Germany, thus it was important to pay attention closely to the language: language purism was a care of the national heritage.
\end{abstract}

Keywords: language purism; 19th century; gymnastic language; Friedrich Ludwig Jahn

Schlüsselwörter: Sprachpurismus; 19. Jahrhundert; Turnsprache; Friedrich Ludwig Jahn

Subject-Affiliation in New CEEOL: Language and Literature - Theoretical Linguistics - Historical Linguistics

DOI: $10.36007 /$ eruedu.2020.3.074-082

\section{Einführung}

Die Leibeserziehung war und ist ein überzeitliches Thema in Deutschland, dem wegen der geschichtlichen Aspekte bis heute viel Aufmerksamkeit gewidmet wird. Das deutsche Turnen ist mit der deutschen Turnsprache schon von Anfang an innig verbunden. Die Wortschöpfungen von Friedrich Ludwig Jahn hielten sich in der Turnsprache und wurden zu einem wesentlichen Teil in den allgemeinen Sprachgebrauch übernommen. Das Turnen und die Turnsprache sind sehr eng mit der deutschen Kultur, Geschichte und Politik verbunden. Diese Sportdisziplin hat ihren historischen Ursprung im deutschsprachigen Raum und ist in deutschsprachigen Ländern sehr beliebt. Dies ist Ansatz zu einer ganzen Reihe sprachlicher, geschichtlicher und soziokultureller Fragestellungen. Kann man in Bezug auf die Turnsprache über Sprachpurismus sprechen? Was hielt Jahn über die Sprachpflege? Welche Ziele und Anforderungen setzte Jahn sich selbst? Diese Fragen bilden den Ausgangspunkt unserer Überlegungen. 


\section{Turnen und die Jahnsche Turnsprache}

Die Turnbewegung war ein Teil der um etwa 1800 entstehenden deutschen Nationalbewegung und spielte vor allem ab 1840 bei der Ausbildung eines gesellschaftlichen Nationalismus in Deutschland eine wichtige Rolle. Sie sollte sowohl Körper als auch Geist harmonisch gestalten, war aber vor allem Mittel zum Zweck. Einerseits sollte sie eine gewaltsame Beseitigung der Napoleonischen Fremdherrschaft bilden und andererseits sollte sie das Bewusstsein beeinflussen. Als Ziel wurde die Schaffung eines deutschen Nationalstaates gesehen (vgl. Röthig 1992, 534-535). In den Jahren 1806 und 1807 stand Deutschland an einem Wendepunkt seiner nationalen Entwicklung. Mit dieser Zeit assoziiert man auch die Entstehung der Turnbewegung in Deutschland, die ihren Anfang im 19. Jahrhundert in der sogenannten Reichsgründungszeit hatte. Diese Bewegung steht in enger Verbindung mit ihrem als Turnvater bekannten Begründer Friedrich Ludwig Jahn (1778-1851), der zur Zeit der Befreiungskriege in Berlin als Organisator einer Turngesellschaft wirkte.

Auf diese Weise hat er zur Entwicklung der Leibesübungen und des politischen und öffentlichen Vereinswesens beigetragen, deren Ursprünge im 18. Jahrhundert bei Johann Christoph GutsMuths liegen. Johann GutsMuths hat eine pädagogisch begründete Gymnastik entwickelt, die weltweit rezipiert wurde und deren Ziel war, die männlichen Schüler zu vernunftgeleiteten Bürgern zu erziehen. Da sich Vernunft nur durch Handeln und Erkenntnis, das heißt nur durch körperliche Aktivitäten und sinnliche Wahrnehmungen, entwickeln könne, hielten die Philanthropen Leibesübungen für unverzichtbar.

Turnen spielte also im 19. Jahrhundert in der Geschichte Deutschlands eine sehr wichtige Rolle und wurde zur Erziehung des Volkes benutzt. Michael Krüger bezeichnete die Turnbewegung daher als eine besondere und symbolische Form des deutschen Nationalismus (vgl. François 2001). Sehr wichtig für die Entwicklung einer bürgerlichen Gesellschaft in Deutschland waren im 19. Jahrhundert auch die von der frühen Turnbewegung unter dem Einfluss Jahns entwickelten Leitbilder und Verhaltensnormen. Dazu gehören die turnerische Haltung, d.h. Selbstdisziplin, die in den „Turngesetzen“ verankerte „turnerische Tugend“, die einheitliche Leinen-Gleichtracht, das Turner-,,Du“, die dem Streben nach einer egalitären Gesellschaft verpflichteten Anschauungen von der Gleichheit vor dem Gesetz. Im Vorbericht seines Buches schrieb Jahn selbst „Von Anfang an zeugte die Turnkunst einen großen Gemeingeist und vaterländischen Sinn, Beharrlichkeit und Selbstverleugnung. Alle und jede Erweiterung und Entwicklung galt gleich als Gemeingut" (Jahn-Eiselen, 1816, 11-12). Die gesellschaftlichen Ziele der Turnbewegung waren ihm nicht weniger bedeutend als die politischen.

Turnen kann als ein nationales Kommunikationssystem bezeichnet werden, das sich in Haltung und Bewegung verkörpert und zahlreiche Symbole zur Verstärkung des Wir -Gefühls entwickelt hat (vgl. Pfister, 2001, 202-220). Das Turnen vertritt die Interessen der ganzen Nation und ist politisch gesinnt. Unter dem Begriff „Kommunikationssystem“ wird hier eine Form des gemeinsamen Lebens, ein Aus- 
druck einer Volksbewegung, getragen von dem Freiheitsgefühl und vom Gefühl der Selbsttätigkeit, verstanden. Die Turnbewegung konzentrierte sich einerseits auf Körperübungen und andererseits führte sie zur Verstärkung der Nationalgefühle und Einigkeit. Turnen war sogleich ein Mittel zur Erziehung des Volkes, aller Menschen, die durch gemeinsame Sprache und Kultur miteinander verbunden seien.

Unter dem Kommunikationssystem wird auch die Turnsprache verstanden, die als ein Verständigungsmittel zwischen Turnlehrern und Turnschülern diente und die Kommunikation sowie die Ausübung des Turnens erleichtern sollte. Der Begriff der „Turnsprache” wurde in Deutschland von dem Begründer der Turnbewegung (Turnvater" Friedrich Ludwig Jahn) im 19. Jahrhundert als Bezeichnung für alle Dinge und Umstände (alle Übungen und Gerätschaften), die im Zusammenhang mit Turnen stehen, eingeführt. Dieses Kommunikationssystem wurde als Ausdruck der Volksbewegung verstanden. Jahn als Lehrer und Patriot wollte das Turnen auch über eine verständliche Fachsprache verbreiten. Die Turnsprache bezeichnet er als Kunstsprache, der er Lebendigkeit zuspricht, weil er sie direkt vom Leben herleitet. „Soll eine Kunstsprache lebendig sein, so muß sie aus dem Leben hervorgehn" (Jahn-Eiselen 1816, 26) - schreibt er in der Vorrede seines Werkes.

Man kann daraus also die Schlussfolgerung ziehen, dass er Turnschülern und -lehrern mit dieser Sprache ein leistungsfähiges und zweckmäßiges Kommunikationssystem zur Verfügung stellen wollte, weil Leibeserziehung für ihn als Lehrer wie auch als Patrioten im Zentrum seines Programmes stand. Das Turnen stand neben Politik und Veränderungen in der deutschen Gesellschaft auch im Zusammenhang mit der geistesgeschichtlichen Entwicklung in Deutschland. Daher erlebte das Turnen nach Jahn am Anfang des 19. Jahrhunderts eine weite Verbreitung in ganz Deutschland (vgl. Krüger 1993, 36).

\section{Sprachpurismus im 19. Jahrhundert}

Nach Peter von Polenz spricht man über zwei Epochenmerkmale des 19. Jahrhunderts. Dazu gehören: erstens der Übergang von der feudal-ständischen zur Industriegesellschaft und zweitens der Nationalismus (vgl. Peter von Polenz, 1999). Mit der Erfahrung der französischen Fremdherrschaft und den Befreiungskriegen wird das 19. Jahrhundert durch Ereignisse geprägt, die den deutschen Sprachnationalismus radikalisieren, auf politischer Ebene aber weder zur Nationalstaatsbildung noch zur Liberalisierung und Demokratisierung führen. Es kommt die Zeit, in der sich die enttäuschten Bürger in die Sphäre von Sprache, Literatur, Kunst und Bildung zurückziehen (vgl. Stukenbrock 2005, 241). Während man im 17. und 18. Jahrhundert über das Konstruieren des Nationalsymbols „deutsche Hauptsprache" spricht, ist im 19. Jahrhundert die Rede von Etablierung der Standardsprache (vgl. Stukenbrock 2005, 241).

Den Begriff des deutschen Sprachpurismus oder der deutschen Sprachreinigung erklärt man als Bestrebungen, die deutsche Sprache von Fremdwörtern und Lehnwörtern zu reinigen. Es ist ein Versuch, die Einflüsse anderer Sprachen auf die eigene einzuschränken, indem man die fremde Lexik durch passende deut- 
sche Wörter ersetzt oder ganz neue deutsche Wörter bildet.

In Deutschland betrifft die Bezeichnung Sprachpurismus laut Peter von Polenz die sprachpflegerischen Bemühungen im 17. und 18. Jahrhunder in Hinsicht auf zwei wesentliche Tendenzen. Zum einen stand die Vermeidung von Fremdwörtern und zum anderen die Pflege sowie Etablierung der deutschen Sprache im Fokus dieser Bemühungen (Polenz, 1967). Im 17. und 18. Jahrhundert konzentrierte sich der Sprachpurismus auf die Sprache der Literatur und Wissenschaft, und im 19 Jh. stand die Fremdwortbekämpfung im Mittelpunkt. Das bedeutet, dass die neu erwachten Nationalgefühle auch in der Sprachpflege von Bedeutung waren.

Der Sprachpurismus war natürlich keine exklusiv deutsche Erscheinung, sondern allgemein in den sich herausbildenden Nationen verbreitet. Als Beispiel kann man hier Polen erwähnen. Sowohl in Deutschland als auch in Polen könnte man die Sprache als ein Band bezeichnen, das die Nation vereinigt und zusammenhält. Dies galt insbesondere vor dem Hintergrund einer fehlenden nationalen Staatlichkeit. Das Streben nach Reinheit der Muttersprache war ein Kampf um die eigene Nation und nationale Identität. Der Sprachpurismus wurde also ein Bestandteil eines kulturpolitischen Kampfes um nationale Selbständigkeit. Diese Erscheinung war mit bestimmten politischen Fakten verbunden. In Deutschland war es vor allem die napoleonische Herrschaft zu Beginn des 19. Jahrhunderts, die zur Ablehnung der französischen Spracheinflüsse führte. Polen war (vom Ende des 18. Jahrhunderts bis 1918) unter die drei Besatzungsmächte Preußen, Russland und Österreich geteilt. Es gab keinen selbständigen polnischen Staat, dieser wurde erst angestrebt. Dies führte zum Erwachen des Nationalgefühls und zur Abgrenzung gegenüber den Besatzungsmächten (Schiewe-Lipczuk-Nerlicki-Westphal 2010, 13).

Die Bemühungen innerhalb der langen Tradition von Sprachpflegevereinen hatten in der Regel neben der Leidenschaft zur deutschen Muttersprache im 19. und Anfang des 20. Jahrhunderts auch eine mehr oder weniger national ausgeprägte Haltung als Hintergrund. Der Sprachpurismus des 19. Jahrhunderts kann also unter nationalen Gesichtspunkten verstanden werden. Deutschland ist ein Land, welches in besonderem Maße und über Jahrhunderte hinweg vor dem Problem der Zusammenführung der Begriffe Sprache, Kultur und Nation stand. Deutsche Intellektuelle nahmen dabei einen maßgeblichen Einfluss auf diese Diskurse innerhalb der ,verspäteten Nation“. Diese Bewegung beeinflusste in Deutschland nicht nur Sprachwissenschaftler, sondern auch verschiedene Interessenvertreter und Interessengruppen, die sich oft aus patriotischen Gründen für den Sprachpurismus eingesetzt hatten, (vgl. Fettig 2009, 6).

Im 19. Jahrhundert wurde die Tradition der Sprachpflege und des Fremdwortpurismus nicht abgerissen, was die Gründung von verschiedenen Vereinen und Gesellschaften bestätigt, die gegen das Fremde kämpften und bei denen die deutsche Sprache und nationale Identität im Mittelpunkt standen. Im Jahr 1814 wurde von Karl C.F. Krause und Christian H. Wolke die „Berlinische Gesellschaft für deutsche Sprache“ gegründet (vgl. Schmidt 1983, 278-289). Danach wurde im Jahre 1817 von Georg F. Grotefend der „Frankfurtische Gelehrtenverein für deutsche Sprache“ ins Leben gerufen (Grotefend 1818, 9-11). Der nächste Schritt des so- 
genannten Kampfes gegen Fremdwörter war die Gründung des „Vereins für deutsche Reinsprache“ von Josef D.C. Brugger im Jahre 1848 in Heidelberg (Brugger 1862, 9). Historisch gesehen, sind diese Ereignisse mit den Napoleonischen Kriegen eng verbunden. In dieser Zeit, d.h. im 19. Jahrhundert wurde der Einfluss des Fremden als existenzielle Gefährdung der nationalen Identität betrachtet. Als wichtigstes Anliegen der sprachpflegerischen Arbeit im behandelten Zeitabschnitt wird durchweg die Bekämpfung des Fremdwortes herausgestellt, die vor allem mit nationalen Motiven begründet wird: Die Überflutung der deutschen Sprache mit fremdem Wortgut wird als geistige Sklaverei und als Ursache für die Beeinträchtigung des Nationalgefühls und Volksbewusstseins gewertet. Die reale oder zumindest so empfundene Volksbedrohung und Sprachbedrohung durch die französische Fremdherrschaft führte zum Nachdenken und zur Reflexion des Individuums und Bewusstseinszunahme des Volkes. Die nationale Identität und Muttersprache gewannen sukzessiv an Bedeutung. All dies führte zur Verstärkung der Nationalgefühle. In einem weiteren Sinn spricht man über den Nationalgeist, Volksnationalgeist und den sprachnationalistischen Diskurs (vgl. Stukenbrock 2005, 246). Alle Menschen wollten ihre Zukunft in eigene Hände nehmen, um ihr Leben selbst zu gestalten. In dieser wichtigen Zeit entdeckten die Völker ihre nationale Identität.

\section{Zu den sprachpuristischen Bestrebungen Jahns im 19. Jahrhundert}

Der Sprachpurismus war im 19. Jh. auch mit der Idee der Einheit Deutschlands verbunden. Diese Stellung und die sprachpuristischen Bestrebungen sind bei Friedrich Ludwig Jahn, bei dem von ihm geschaffenen Turnen und der zugehörigen Turnsprache genau zu sehen. In seinem Werk „Die deutsche Turnkunst“ gab Jahn die Grundlage für die Bildung der Turnsprache und für die Systematik der Turnübungen. Im Vorbericht dieses Werkes beschreibt er sehr präzis die Rolle und Funktion des Turnens und der Turnsprache und stellt fest, welchen Voraussetzungen und Regeln die Turnsprache entsprechen soll und wie ein neues Wort aus der Turnsprache gestaltet werden muss. Jahn hat die Regeln konstruiert und bei der Bildung der Turnterminologie eingehalten. Die Jahnschen Voraussetzungen sind untereinander vereinbar. Die Hauptsache war für Jahn jedoch, dass die Wörter aus der deutschen Sprache kamen.

Schon seine erste Forderung der Bildungsregeln schloss den Gebrauch von Fremdwörtern nahezu aus: „eine Deutsche Wurzel sein, oder nachweislich von einer solchen stammen" (Jahn-Eiselen 1816, 50) Jahn hat zum Beispiel das französische Wort echappés mit der deutschen Bezeichnung Hocke ersetzt. Im weiteren Teil des Textes wird näher auf andere Beispiele hingewiesen.

Die Erziehung sah Jahn nicht nur in körperlicher Tätigkeit, sondern wies dabei auch auf eine entscheidende Rolle der deutschen Sprache als Muttersprache hin. Die Sprache war für inn ein wichtiges Mittel zum Zweck der nationalen Einheit, und er schrieb ihr eine Schlüsselrolle zu. Hieraus ergibt sich der Jahnsche Sprachpu- 
rismus. Im Vorbericht zu seinem Werk „Die deutsche Turnkunst“ (Jahn-Eiselen 1816) weist Jahn auf die Wichtigkeit des Turnens und der Turnsprache sowie auf die sprachpuristischen Bestrebungen hin. Ausgangspunkt der sprachpuristischen Bestrebungen wurden aber die Turnplätze. Es muss jedoch angedeutet werden, dass Jahn anfangs nicht überall auf Zustimmung stieß. Es gab offenbar Zweifel und Spott, worauf Jahn auch im Vorbericht seines Werkes „Die deutsche Turnkunst" hinweist.

\begin{abstract}
„Doch sonderte sich ein Kern aus, der auch im Winter als Stamm zusammenhielt, und mit dem dann im Frühjahr 1811 der erste Turnplatz in der Hasenheide eröffnet wurde. Jetzt wurden im Freien, öffentlich und vor jedermanns Augen von Knaben und Jünglingen mancherlei Leibesübungen unter dem Namen Turnkunst in Gesellschaft getrieben. Damals kamen die Benennungen Turnkunst, turnen, Turner, Turnplatz und ähnliche miteinander zugleich auf. Das gab nun bald ein gewaltig Gelaufe, Geschwatz und Geschreibe. Selbst durch französische Tagblätter mußte die Sache Gassen laufen. Aber auch hier zu Lande hieß es anfangs: Eine neue Narrheit, die alte Deutschheit wieder aufbringen wollen. Dabei blieb es nicht. Vorurtheile wie Sand am Meer wurden von Zeit zu Zeit ruchtbar. Sie haben bekanntlich niemals vernünftigen Grund, mithin wäre es lächerlich gewesen, da mit Worten zu widerlegen, wo das Werk deutlicher sprach" (Jahn-Eiselen 1816, 10).
\end{abstract}

Auf diese Art und Weise konnte Jahn im Bereich der Turnsprache seine Sprachreform konsequent realisieren. „Da die meisten Geräte und Übungen völlig innovativ waren, lag bereits 1812 eine von Jahn konstruierte, reiche Fachsprache des Turnens vor, die nahezu ohne Fremd- und Lehnwörter auskam und weitestgehend noch heute von Turnern so genutzt wird“" (Lotze 2009, 245).

Es kann also festgestellt werden, dass Jahn als Patriot mit dem Fremden sowohl bei dem Entstehen der deutschen Turnbewegung als auch bei der Bildung der Turnsprache kämpfte. Jahn verstand unter Fremden Franzosen und Fremdwörter. Er kämpfte gegen die französische Herrschaft, gegen Napoleon und gegen die fremde Sprache d.h. gegen französische Fremdwörter.

Schon in dem Wort turn weist er darauf hin, dass er turn- für ein deutsches Urwort hält und das französische tourner als Entlehnung aus dem Deutschen ausgibt. In seinem Werk ,Die deutsche Turnkunst“ betont Jahn bezüglich der Turnsprache ein „unbestrittenes Recht, eine deutsche Sache in deutscher Sprache, ein deutsches Werk mit deutschem Wort zu benennen" (vgl. Jahn-Eiselen 1816, 25). Und im weiteren Teil schreibt er „Warum auch bei fremden Sprachen betteln gehen und im Ausland auf Leih und Borg nehmen, was man im Vaterland reichlich und besser hat"(vgl. Jahn-Eiselen1816, 25). Er schätzte die deutsche Sprache sehr und schrieb ihr vielseitigere Ausdrucksmöglichkeiten als anderen Sprachen zu. Da die Turnsprache für ein Volk mit vaterländischer Gesinnung gebildet wurde, um zur Einheit Deutschlands beizutragen, stand die Sprachreinigung vom Fremden an der ersten Stelle. Mit der Sprachreinigung war die geistige Erneuerung verbunden. Indem man die Sprache reinigte, konnte man auch das Denken von fremden Einflüssen befreien.

An einigen Beispielen lässt sich Jahns sprachpuristische Arbeit zu zeigen. Wir 
können hier das Wort voltigieren erwähnen. Es stammt ursprünglich aus dem Französischen und besitzt keine deutsche Wurzel (vgl. Meyers Großes Konversations-Lexikon 1909, 254) Um es zu vermeiden, führt Jahn statt voltigieren das Wort schwingen in die Turnsprache ein. Neben voltigieren kann man auch andere Vorübungen erwähnen, die ursprünglich mit Namen französischer Herkunft belegt waren. Man findet also zum Beispiel das Wort Croisé, das bei Jahn Schere heißt. Im Weiteren wird der Begriff Echappé mit Hocke ersetzt. Neben den Namen Ecarté und Ecarquillade (GutsMuths 1804, 224-226) findet man die deutschen Bezeichnungen Kreuzsprung und Grät- oder Grätschsprung.

Als andere Beispiele kann man die Übungsbezeichnung Pirouette erwähnen. GutsMuths verwendet dieses Wort, aber nennt auch diese Übung Drehen. Bei Jahn steht Drehung. Dann haben wir statt des französischen Balance das deutsche Wort Schweben. Die Tabelle unten veranschaulicht Beispiele der Jahnschen Verdeutschungen der Turnsprache.

\begin{tabular}{|l|l|}
\hline Die französischen Bezeichnungen & $\begin{array}{l}\text { Die Entsprechungen der französischen } \\
\text { Bezeichnungen im Deutschen }\end{array}$ \\
\hline Echappés & Hocke \\
\hline Pommaden & Sattel \\
\hline Volten & Drehung/ Kreis \\
\hline Ecartés /Ecarquillade & Kreuzsprung / Grät- od. Grätschsprung \\
\hline Revers & Kehre \\
\hline Croisés & Schere \\
\hline Pirouette & Drehung \\
\hline Balance & Schweben \\
\hline Ricochettieren & Gellen \\
\hline
\end{tabular}

Tabelle 1. Die Zusammenstellung der französischen Bezeichnungen und der Jahnschen Verdeutschungen der Turnsprache nach GutsMuts (1804), Jahn-Eiselen (1816).

Weiterhin schrieb er über die deutsche Sprache Folgendes „Nimmer mehr wird die deutsche Sprache eine Mangsprache werden“ (Jahn-Eiselen 1816, 27) und weiter ,Ihr ist Wortmengerei - Armuth, Reinheit - Reichthum und Reinigung - Bereicherung. Die Fremdsucht ist ihr Galle, Gift und Greuel, ein Irrleuchten im Dämmer und Nebel." (Jahn-Eiselen 1816, 27 -28) Jahn betrachtete den Einfluss des Fremden als existenzielle Bedrohung für die nationale Identität und kritisierte einfach alles, was fremd war, und bezeichnete es als etwas Giftiges, als eine Gefährdung für das Volk und für die deutsche Nation. Sehr kritisch war Jahn auch zu den Benutzern von Fremdwörtern eingestellt. Er bezeichnete sie als „Worttäuscher“ und „Wortbeschwörer" die, ,,ihrer Neusucht fröhnen“ und damit „,hochverräterisch“ das deutsche Wesen preisgeben (Jahn 1810, 334). Seine Einstellung war sehr radikal, und er selbst war intolerant gegenüber allen Fremdwortbenutzern. 


\section{Fazit}

Die Turnsprache hat sich im Laufe der Zeit mit der Gesellschaft verändert. Sie musste neuen Voraussetzungen und Anforderungen der Gesellschaft und den Veränderungen der politischen Bedingungen angepasst werden. Dies sieht man bei den Jahnschen sprachpuristischen Bestrebungen, die eng mit der Politik verbunden waren. Dabei ging es darum, die Turnsprache von Fremdwörtern zu reinigen und sie aus Wörtern deutscher Herkunft zu bilden oder das Fremdsprachige einfach durch den deutschen Wortschatz zu ersetzen. Die Französische Revolution, der Zusammenbruch des Alten Reiches und die französische Besetzung deutschsprachiger Territorien förderten die Politisierung der Sprachreinigung im Sinne des den alten Kulturpatriotismus allmählich ablösenden deutschen Nationalismus, der ein Sprachnationalismus mit primärer Symbolfunktion von Sprache war. In der Zeit des „nationalen Aufbruchs“ gegen Napoleon blieb der Fremdwortpurismus zwar ohne großes Echo in der Öffentlichkeit, er wurde aber in akademisch gebildeten Kreisen als ein sprachwissenschaftliches Thema sehr intensiv diskutiert (vgl. Polenz von Peter 1999, 266-26). Der deutsche Wortschatz war nach Ansicht der Puristen eine Garantie für die nationale Identität und Sprachidentität, deswegen wollte Jahn als Patriot die Sprache von Fremdwörtern reinigen. Die Sprache verstand man als Garanten der Volkszugehörigkeit. Zusammenfassend kann festgestellt werden, dass die sprachpuristischen Motive und Bestrebungen bei Friedrich Ludwig Jahn und von inm geschaffenen Turnen und seiner Turnsprache genau zu sehen sind. Für Jahn als Turnvater, Spracherzieher und Patriot standen Nationalgefühl und gesellschaftliche Erneuerung im Zuge der Bildung und Ausbreitung der Turnbewegung obenan. Es wird deutlich, wie stark Turnen und Turnsprache Mittel zum Zweck waren, die nationale Identität zu erwirken.

In Bezug auf die gegenwärtige deutsche Sprache fühlt sich kaum jemand einem Sprachpurismus verpflichtet. Dieser Begriff ist heute ein Anachronismus. Die Bemühungen um fremdwortarmes Deutsch werden eher der Sprachkritik und Sprachpflege zugeordnet, denen sich mehrere Sprachvereine widmen. Sprachpflegevereine wenden sich gegen die Verwendung von Anglizismen und versuchen diese durch deutsche Wörter und Bezeichnungen zu ersetzen. In Deutschland beschäftigt sich mit diesem Thema der Verein Deutsche Sprache. Zu diesem Zweck werden auch Verdeutschungswörterbücher angeboten. In manchen Sportdisziplinen, wie z.B. im Fußball, findet man heute eine groß e Zahl an Fremdwörtern, vor allem aus dem Englischen wie zum Beispiel Keeper als Bezeichnung für Torwart, oder Coach als Bezeichnung für Trainer (vgl. Timm 2005, 101, 278) 


\section{Literaturverzeichnis}

Brugger, Joseph Dominic Carl (1862): Geschichte der Gründung und Entwicklung des Vereins der deutschen Reinsprache. Heidelberg: University of Michigan Library.

Fettig, Daniela (2009): Sprachpurismus und Internationalismen. Studienarbeit. München: GRIN Verlag.

François, Etienne (2001): Deutsche Erinnerungsorte. Bd. 2. München: Beck.

Grotefend, Georg Friedrich (1818): Abhandlungen des frankfurtischen Gelehrtenvereines für deutsche Sprache. Bd.1. Frankfurt/M: Varrentrapp.

GutsMuths, Johann Christoph Friedrich (1804): Gymnastik für die Jugend, enthaltend eine praktische Anweisung zu Leibesübungen. Schnepfenthal: Buchhandlung der Erziehungsanstalt.

Jahn, Friedrich Ludwig-Eiselen, Ernest Wilhelm Bernhard (1816): Die Deutsche Turnkunst zur Einrichtung der Turnplätze. Berlin: Auf Kosten der Herausgeber.

Krüger, Michael (1993): Einführung in die Geschichte der Leibeserziehung und des Sportes. Leibeserziehung im 19. Jahrhundert: Turnen fürs Vaterland. Bd. 2. Schondorf: Hofmann.

Krüger, Michael (2000): Turnen und Turnphilologie des 19. Jahrhunderts als Vorläufer der modernen Sportwissenschaft. Sportwissenschaft. 30/2. 197-210. p.

Lotze, Netaya (2009): Frisch, fromm, fröhlich, frei. Zur Bedeutung einer Sprache des Turnens-von Turnvater Jahn bis heute. In: Flickflack, Foul und Tsuhakara. Bd. 10. Mannheim: Dudenverlag. 244-261. p.

Meyers Gro es Konversations-Lexikon (1909) Band 20., Leipzig.

Polenz, Peter von (1967): Sprachpurismus und Nationalsozialismus. Die ,Fremdwort'-Frage gestern und heute. In Nationalismus in Germanistik und Dichtung. Dokumentation des Germanistentages in München vom 17.-22. Oktober 1966. Wiese, Benno von-Henß, Rudolf (Hrsg.). Berlin: E. Schmidt Verlag.

Polenz, Peter von. (1999): Deutsche Sprachgeschichte von Spätmittelalter bis zur Gegenwart. Bd. 3. 19. und 20. Jahrhundert. Berlin: De Gruyter.

Pfister, Gertrud Ursula (2001): Frisch, fromm, fröhlich, frei. In Deutsche Erinnerungsorte. $B d$. 2. Francois, Etienne-Schulze, H. (Hrsg.). München: Beck.

Röthig, Peter (1992): Sportwissenschaftliches Lexikon. Schorndorf: Hofmann.

Schiewe, Jürgen-Lipczuk, Ryszard-Nerlicki, Krzysztof-Westphal, Werner (2010): Kommunikation für Europa II. Sprache und Identität. Frankfurt/M: Peter Lang.

Schmidt, Hartmut (1983): Die Berlinische Gesellschaft für deutsche Sprache an der Schwelle der germanistischen Sprachwissenschaft. Zeitschrift für Germanistik 4/3, 278-289. p. Stukenbrock, Anja (2005): Sprachnationalismus: Sprachreflexion als Medium kollektiver Identitätsstiftung in Deutschland (1617-1945). Berlin: De Gruyter.

Timm, Friedrich (2005): Das moderne Fremdwörterlexikon: Unbekannte Begriffe schnell verstehen und sicher anwenden. Köln: Naumann \& Göbel. 